\title{
Malocclusion Characteristics as Risk Factors for Temporomandibular Disorders: Lessons Learned from a Meta-Analysis
}

\author{
Claudia L. Cruz, ${ }^{1}$ Kevin C. Lee, ${ }^{1}$ Jae Hyun Park, ${ }^{2,3}$ and Athanasios I. Zavras ${ }^{4}$ \\ ${ }^{1}$ College of Dental Medicine, Columbia University, 622 W. 168th Street, Suite PH17-306, New York, NY 10032, USA \\ ${ }^{2}$ Postgraduate Orthodontic Program, Arizona School of Dentistry \& Oral Health, Mesa, AZ 85206, USA \\ ${ }^{3}$ Graduate School of Dentistry, Kyung Hee University, Seoul, Republic of Korea \\ ${ }^{4}$ Department of Pediatric Dentistry, Boston University, Henry M. Goldman School of Dental Medicine, 100 E. Newton Street, \\ Boston, MA 02118, USA \\ Correspondence should be addressed to Kevin C. Lee; kcl2136@cumc.columbia.edu
}

Received 13 June 2015; Accepted 27 July 2015

Academic Editor: Giulio Gasparini

Copyright (C) 2015 Claudia L. Cruz et al. This is an open access article distributed under the Creative Commons Attribution License, which permits unrestricted use, distribution, and reproduction in any medium, provided the original work is properly cited.

\begin{abstract}
The objective of this meta-analysis was to evaluate the association of various malocclusion characteristics with temporomandibular disorders (TMDs). Two reviewers identified articles through database searches of MEDLINE (Ovid) and LILACS and hand searches of major orthodontic journals and selected references. Random-effects models were used to calculate weighted pooled odds ratio (OR) estimates of malocclusion exposures. Six articles qualified for inclusion in the final quantitative analysis. Our study found that static occlusal factors had no significant association with TMD. Of the dynamic occlusal factors assessed, only the absence of canine guidance, laterotrusive interferences, and retruded contact position to maximal intercuspation slide length $\geq 2 \mathrm{~mm}$ demonstrated significant ORs. These results should be viewed with caution, as reporting biases were difficult to assess, and heterogeneity estimates may have been underestimated due to the limited number of studies within each comparison. TMD is a term that encompasses a broad group of dysfunctions, and meta-analyses should only synthesize studies with similar diagnostic criteria. At the present, there is a paucity of studies available that could be properly synthesized to answer the research question posed. Individual studies have too much variability among their methods, and researchers need to clearly define and state their TMD factor definition.
\end{abstract}

\section{Introduction}

Twenty years ago, a comprehensive literature review [1] of occlusion, orthodontic treatment, and temporomandibular joint disorders (TMDs) spearheaded by McNamara et al. concluded that the association between occlusion and TMD remained minor, with some studies estimating that occlusion contributed to $10-20 \%$ of TMD cases [2,3]. McNamara et al. suggested that orthodontic treatment did not increase or decrease the odds of developing TMD in the future, and, ultimately, their group recommended that clinicians manage TMD with reversible therapies until more definitive results were available. This caution stemmed from the fact that studies evaluated demonstrated what McNamara et al. considered to be "significant methodological weaknesses" [1].
Dworkin and LeResche [4] developed the most comprehensive case definition for TMD, the Research Diagnostic Criteria for Temporomandibular Disorders (RDC/TMD). Since that development, several researchers have attempted to revisit the updated literature and review the interactions of morphological and functional occlusal factors and TMD. With large prospective efforts, like the OPPERA study [5], the research community is moving toward a systemic model of TMD and focusing on the comorbidities. However, within orthodontic community, the concept of TMD as a consequence of malocclusion is still promoted not only in academic circles, but also clinically $[6,7]$. This disagreement poses a major limitation to both dental clinicians and educators. Within orthodontic training, there does not exist a standardized case-finishing protocol for TMD cases, primarily 
because there is no standardized curriculum or consensus school of thought [8].

Since the introduction of the RDC/TMD diagnostic criteria, researchers have begun making efforts to standardize their case definitions of TMD, and as a result it is of clinical interest to revisit the associations of malocclusion characteristics with TMD given the improved materials and methods of TMD research over the past twenty years. This study sought to overcome the methodical weaknesses and heterogeneity of previously published analyses by only synthesizing studies with similar TMD case definitions. It is hypothesized that studies conducted with a similar case definition of TMD would demonstrate associations with similar malocclusion exposures.

\section{Methods and Procedures}

2.1. Search Strategies. The search strategy for this review was designed with the assistance of a specialized health-sciences librarian. Electronic database searches were conducted with Medical Subject Headings, keywords, and keyword combinations. Electronic search criteria included malocclusion and TMD and temporomandibular joint (TMJ) search terms. Appropriate truncations and word combinations were used in each search. The literature review spanned from the inception of the database until June 2014 on an electronic search of MEDLINE (Ovid) and LILACS. The hand search was limited to four journals (American Journal of Orthodontics and Dentofacial Orthopedics, Angle Orthodontist, European Journal of Orthodontics, and Journal of Dental Research) from 1990 through 2014, and selected references were inspected to locate other relevant articles. Electronic, hand, and reference results were cross-referenced in EndNote to eliminate duplicate studies. Papers in a language other than English were included only if they could be translated.

Two independent reviewers screened the results of the searches. The first screening of references was performed using the titles, and the second screening was performed using abstracts. Abstracts identified as useful were coded for potential inclusion. When the reviewers disagreed about classification during the second screening, reasons were identified and discussed until a consensus could be reached. Full-text articles were retrieved when both reviewers felt that the articles potentially met the inclusion criteria. The full texts were read and then ultimately included or excluded based upon how well the studies met the inclusion/exclusion criteria. Data were extracted from all included studies into a spreadsheet.

2.2. Inclusion/Exclusion Criteria. This review aimed to include randomized control studies (RCTs), cohort studies, and case/control studies conducted with adults populations that defined TMD as a dysfunction of myofascial pain with the assistance of a clinical examination. Ultimately, it was decided that case/control studies would be used given the dearth of RCTs and cohort studies on malocclusion and TMD. Studies evaluating osteoarthritis and genetic disorders as generalized joint hypermobility as well as studies only evaluating the effects of occlusal trauma or parafunctions on the TMJ were excluded. The population was limited to humans. Since the TMD treatment needs in children and adolescents have been reported to be only $2-4 \%$ in a given population, this study only focused on adult participants with clinically relevant TMD. For all included case/control studies, the exposures were malocclusion, and the disease or outcome was TMD. The authors conducted a meta-analysis and calculated a weighted pooled odds ratio (OR) estimate when two or more of the included studies evaluated the same malocclusion exposure.

\section{Results}

Figure 1 outlines the results of the search process that culminated in 6 studies being selected for final quantitative analysis. The electronic search identified a total of 815 publications and the hand search identified an additional 361 publications. 57 studies from the electronic search and 18 studies from the hand search evaluated the association between malocclusion and TMD. Only 73 articles were retrieved for full-text review, as 2 articles from the LILACS search were unavailable in full text. The retrieved full-text publications were read, and 10 articles met the requirements of the inclusion criteria by consensus between authors. From these articles, the investigators listed all of the malocclusion risk factors that had been determined to have influenced the development of TMD. An assessment of the retrieved articles was performed based on 8 preestablished characteristics (Table 1).

For the quantitative analysis, 3 studies [16-18] were excluded because of inadequate statistics and 2 of them [16, 17] only presented adjusted odds ratios (OR). For those 2 studies, both authors were contacted for unadjusted ORs, but neither was able to retrieve his/her original dataset. One of the excluded studies [18], in addition to having inadequate statistics, incidentally did not evaluate TMD as an outcome and had a small sample size. Withdrawals (dropouts) were declared in 3 studies $[12,13,17]$, and it is possible that the dropouts affected the study group characteristics. Three studies explicitly mentioned the use of blinded examiners $[11,12$, $14,16]$. Intrarater or interrater calibration and measurement reproducibility were assessed in 6 studies $[9,11-13,16,18]$, and of the remaining 4 studies, all used a single trained operator $[10,14,15,17]$.

Some attempts were made in 7 studies [9-11, 13, 14, 17, 18] to match cases and controls, but none of these efforts proved satisfactory, as there were no cases where more than 2 characteristics matched the controls. Of those studies that did attempt to match study groups, 3 studies $[9,10,14]$ controlled for gender through inclusion criteria by exclusively enrolling female subjects. Three studies [14, 16, 17] controlled for selected post hoc confounders by adjusting odds ratios; however, adjusted odds ratios were not considered in this analysis $[14,16,17]$. A total of 6 studies addressing the association of occlusal factors with TMD were ultimately included in the analysis (Table 2) [9-14]. ORs of malocclusion exposures were recorded for each included study (Table 3 ). Mohlin et al's work [13] was included despite the absence of a formal Research Diagnostic Criteria for Temporomandibular Disorders (RDC/TMD) diagnostic criterion because each 


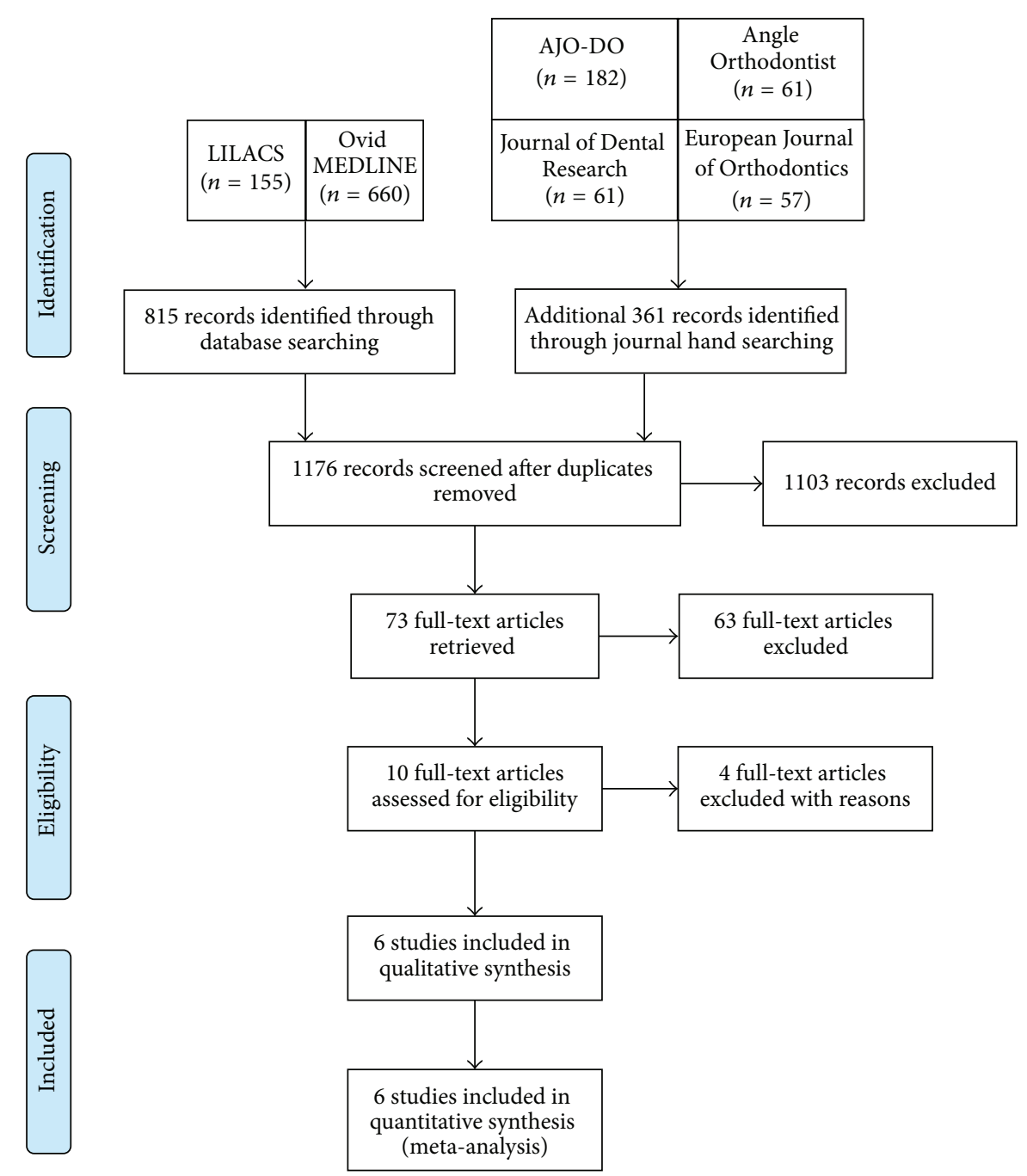

FIGURE 1: Flow chart describing systematic research search and study selection process.

TMD diagnosis was conducted with a clinical examination and the criteria appeared to align with those of the RDC/TMD Axis 1 group 1 diagnostic algorithm. Costa et al.s study [15] was excluded despite adequate data and casecontrol design because the Fonseca questionnaires used for TMD diagnoses were conducted through interviews and selfreports without clinical examinations.

3.1. Characteristics of the Included Studies. The individual sample sizes (Table 2) of the studies ranged from 102 to 1018 subjects with three of the studies enrolling only female subjects. All included studies had diagnosed TMD cases with the aid of a clinical examination. Within a given study, the same trained operator(s) conducted the clinical evaluation for occlusal factors, but only two studies $[13,14]$ mentioned operator blinding. Four studies were guided by an Axis 1 RDC/TMD classification, with one study [12] including the Axis $1 \mathrm{RDC} / \mathrm{TMD}$ criteria as only one among many other possible indications for TMD diagnosis. Three studies [9, 10, 12] defined TMD exclusively as a muscular disorder.
Of the remaining three studies, one [14] defined TMD by an RDC/TMD classification without specifying the axis, another defined TMD by an Axis I RDC/TMD without specifying the group [11], and the third defined TMD from a list of five indications that included both muscular and joint dysfunction [13]. One study used a 2-wave longitudinal cohort design deriving both controls and cases from the same sample of respondents with complete follow-up data [12].

3.2. Quantitative Results of the Meta-Analysis. This study evaluated the association between the presence of occlusal variables and the diagnosis of TMD. A random-effects model meta-analysis of two studies resulted in an overall pooled odds ratio of laterotrusive interferences of 2.190 (95\% CI [1.259, 3.808], $p=0.005$, and $I^{2}=0 \%$ ) (Figure 2(a)), which suggested that the presence of working side interferences increased the odds of TMD diagnosis. This effect was highly significant. Likewise, a similar analysis of the overall pooled odds ratio for patients without canine guidance demonstrated that the absence of canine guidance significantly increased 


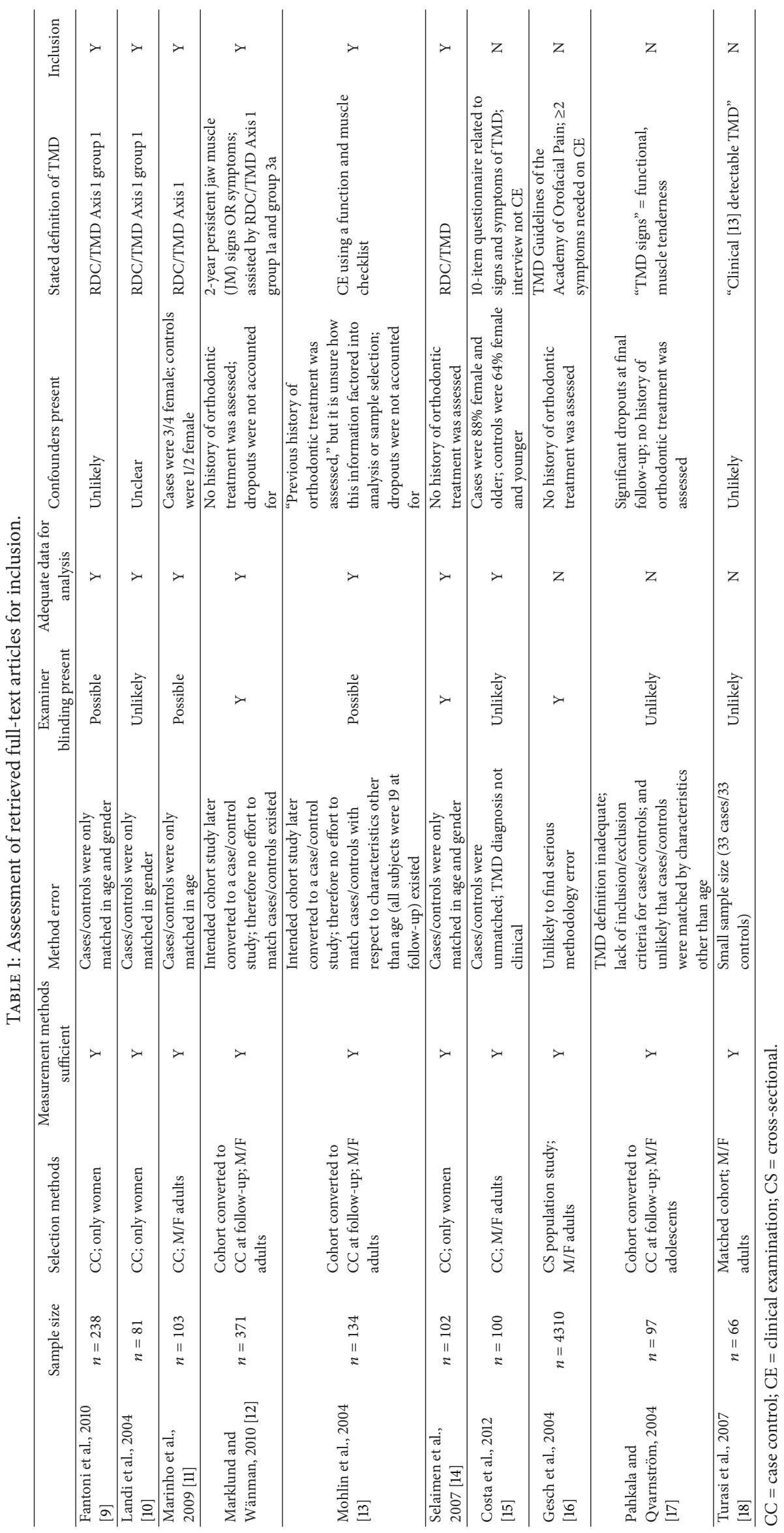




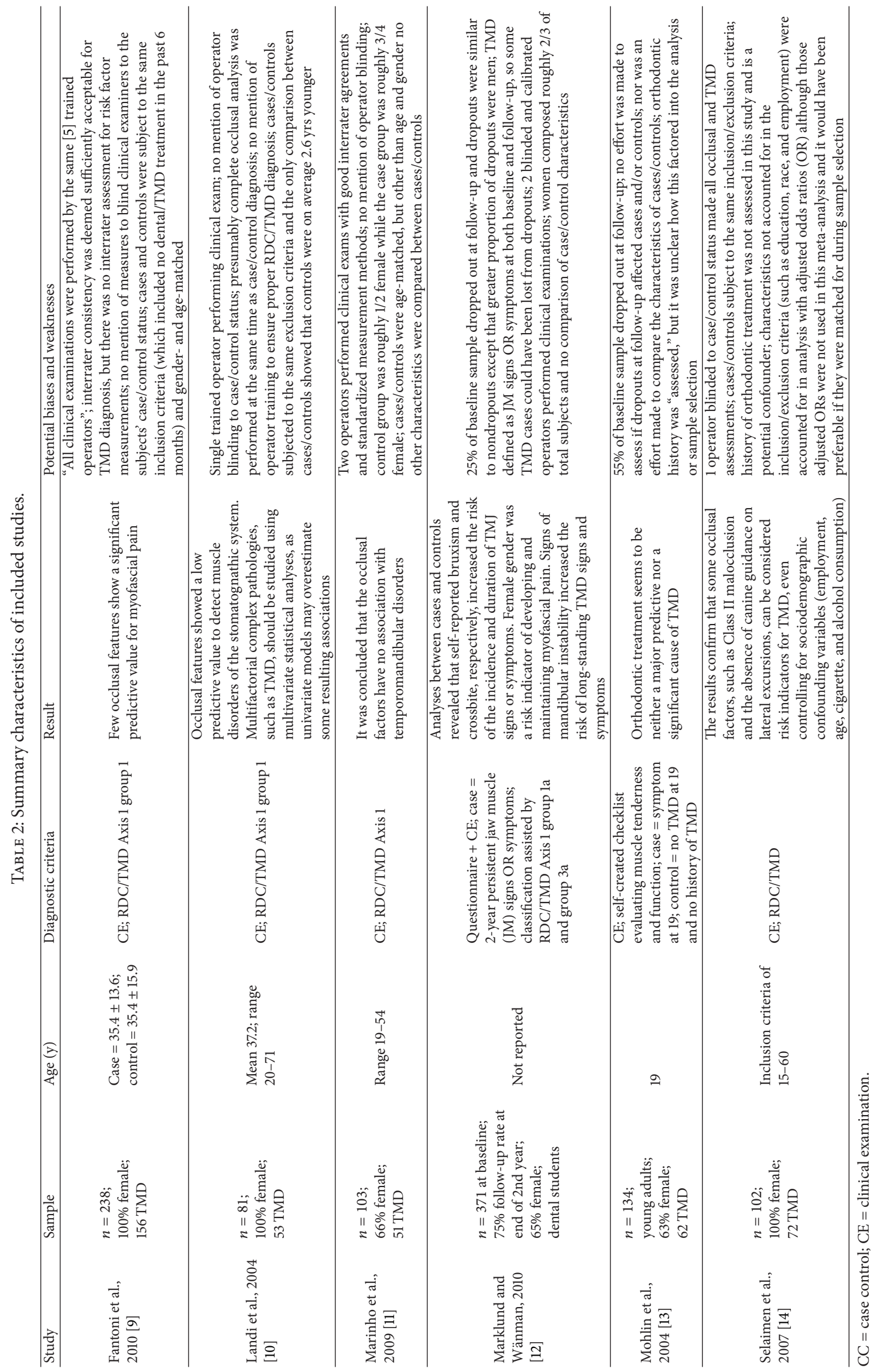




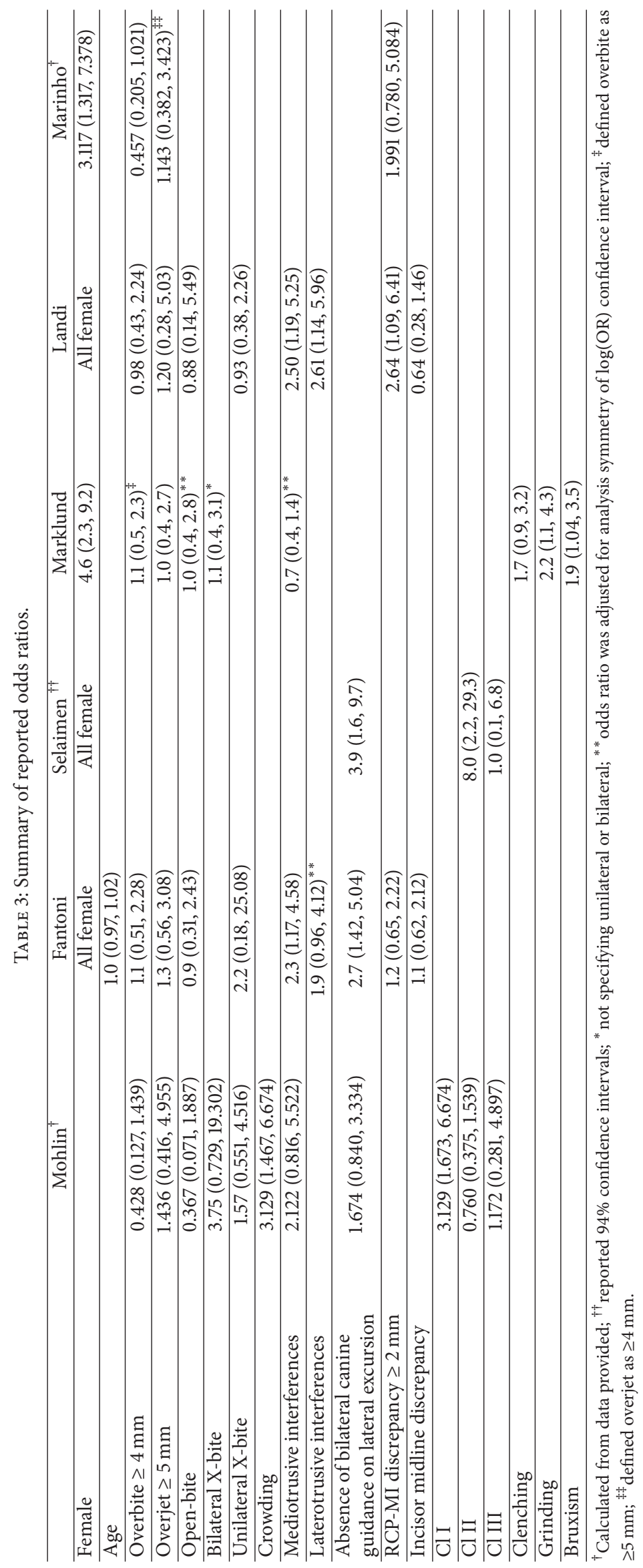




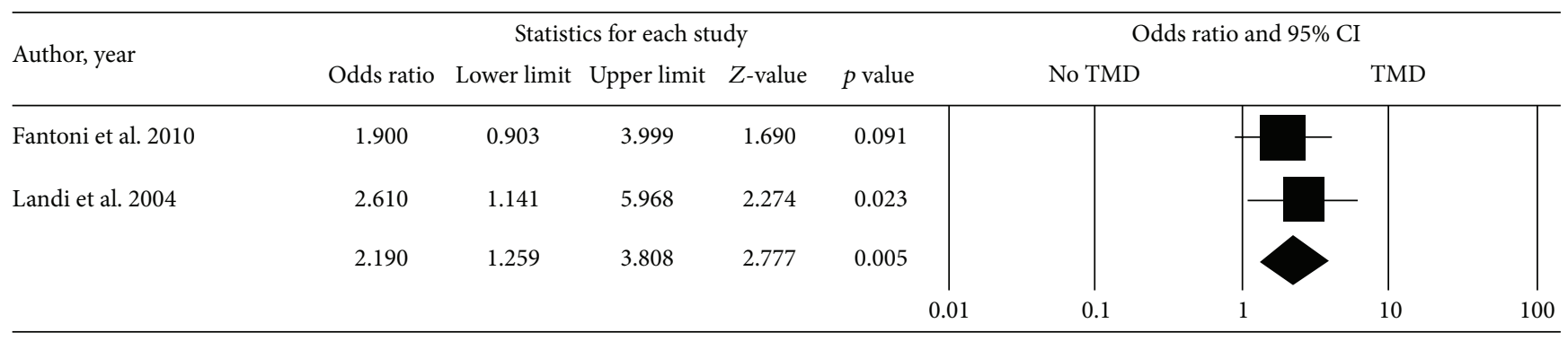

(a)

\begin{tabular}{|c|c|c|c|c|c|c|c|c|c|}
\hline \multirow{2}{*}{ Author, year } & \multicolumn{5}{|c|}{ Statistics for each study } & \multicolumn{4}{|c|}{ Odds ratio and 95\% CI } \\
\hline & Odds ratio & Lower limit & Upper limit & $Z$-value & $p$ value & & No TMD & TMD & \\
\hline Fantoni et al. 2010 & 2.700 & 1.433 & 5.087 & 3.074 & 0.002 & & & . & \\
\hline Mohlin et al. 2004 & 1.674 & 0.840 & 3.334 & 1.465 & 0.143 & & & & \\
\hline \multirow[t]{3}{*}{ Selaimen et al. 2007} & 3.900 & 1.525 & 9.974 & 2.841 & 0.005 & & & & \\
\hline & 2.446 & 1.573 & 3.802 & 3.972 & 0.000 & & & & \\
\hline & & & & & & 0.01 & 0.1 & 10 & 100 \\
\hline
\end{tabular}

(b)

\begin{tabular}{|c|c|c|c|c|c|c|c|c|}
\hline \multirow{3}{*}{$\begin{array}{l}\text { Author, year } \\
\text { Fantoni et al. } 2010\end{array}$} & \multicolumn{5}{|c|}{ Statistics for each study } & \multicolumn{2}{|c|}{ Odds ratio and $95 \% \mathrm{CI}$} & \\
\hline & \multirow{2}{*}{$\begin{array}{c}\text { Odds ratio } \\
1.200\end{array}$} & \multirow{2}{*}{$\frac{\text { Lower limit }}{0.649}$} & \multirow{2}{*}{$\frac{\text { Upper limit }}{2.218}$} & \multirow{2}{*}{$\frac{Z \text {-value }}{0.582}$} & \multirow{2}{*}{$\frac{p \text { value }}{0.561}$} & No TMD & \multicolumn{2}{|l|}{ TMD } \\
\hline & & & & & & & & \\
\hline Landi et al. 2004 & 2.640 & 1.089 & 6.402 & 2.148 & 0.032 & & & \\
\hline \multirow[t]{3}{*}{ Marinho et al. 2009} & 1.991 & 0.780 & 5.084 & 1.439 & 0.150 & & & \\
\hline & 1.668 & 1.032 & 2.695 & 2.089 & 0.037 & & & \\
\hline & & & & & & 0.1 & 10 & 100 \\
\hline
\end{tabular}

(c)

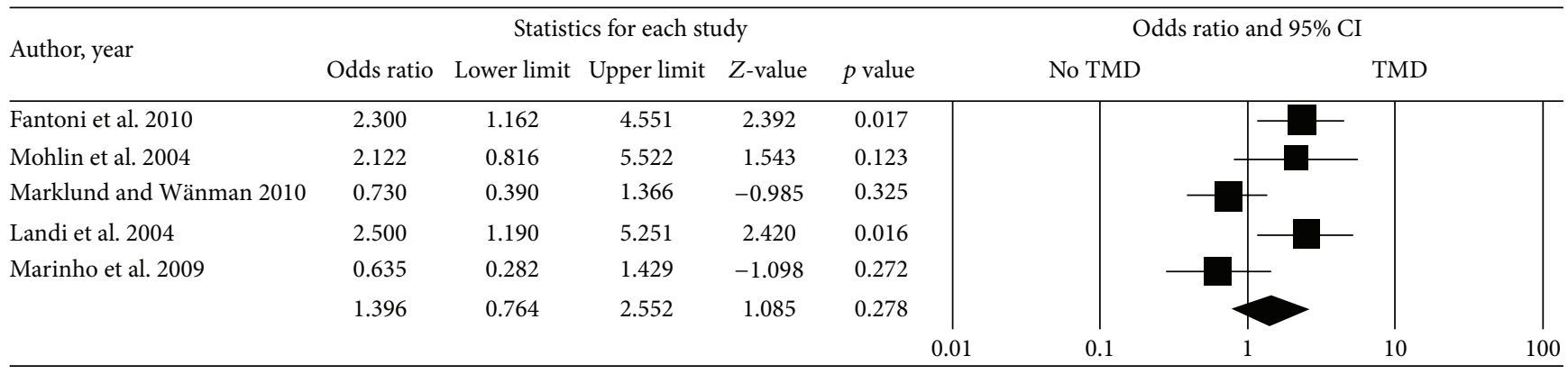

(d)

\begin{tabular}{|c|c|c|c|c|c|c|c|c|}
\hline \multirow{2}{*}{ Author, year } & \multicolumn{5}{|c|}{ Statistics for each study } & \multicolumn{2}{|c|}{ Odds ratio and $95 \%$ CI } & \\
\hline & Odds ratio & Lower limit & Upper limit & $Z$-value & $p$ value & No TMD & TMD & \\
\hline Fantoni et al. 2010 & 0.900 & 0.321 & 2.520 & -0.201 & 0.841 & & & \\
\hline Mohlin et al. 2004 & 0.367 & 0.071 & 1.887 & -1.200 & 0.230 & & & \\
\hline Marklund and Wänman 2010 & 1.000 & 0.385 & 2.598 & 0.000 & 1.000 & & & \\
\hline \multirow[t]{3}{*}{ Landi et al. 2004} & 0.880 & 0.141 & 5.511 & -0.137 & 0.891 & & & \\
\hline & 0.828 & 0.451 & 1.520 & -0.609 & 0.543 & & & \\
\hline & & & & & 0.01 & 0.1 & 10 & 100 \\
\hline
\end{tabular}

(e)

FIgURE 2: Continued. 


\begin{tabular}{|c|c|c|c|c|c|c|c|c|c|}
\hline \multirow{3}{*}{$\begin{array}{l}\text { Author, year } \\
\text { Fantoni et al. } 2010\end{array}$} & \multicolumn{5}{|c|}{ Statistics for each study } & \multicolumn{4}{|c|}{ Odds ratio and $95 \% \mathrm{CI}$} \\
\hline & \multirow{2}{*}{$\begin{array}{c}\text { Odds ratio } \\
1.100\end{array}$} & \multirow{2}{*}{$\begin{array}{c}\text { Lower limit } \\
0.520\end{array}$} & \multirow{2}{*}{$\begin{array}{c}\text { Upper limit } \\
2.326\end{array}$} & \multirow{2}{*}{$\frac{Z \text {-value }}{0.249}$} & \multirow{2}{*}{$\begin{array}{r}p \text { value } \\
0.803\end{array}$} & & No TMD & \multicolumn{2}{|l|}{ TMD } \\
\hline & & & & & & & & & \\
\hline Mohlin et al. 2004 & 0.428 & 0.127 & 1.439 & -1.372 & 0.170 & & & & \\
\hline Marklund and Wänman 2010 & 1.100 & 0.513 & 2.359 & 0.245 & 0.807 & & & & \\
\hline Landi et al. 2004 & 0.980 & 0.429 & 2.237 & -0.048 & 0.962 & & & & \\
\hline \multirow[t]{3}{*}{ Marinho et al. 2009} & 0.457 & 0.205 & 1.021 & -1.910 & 0.056 & & & & \\
\hline & 0.809 & 0.544 & 1.202 & -1.051 & 0.293 & & & & \\
\hline & & & & & & 0.01 & 0.1 & 10 & 100 \\
\hline
\end{tabular}

(f)

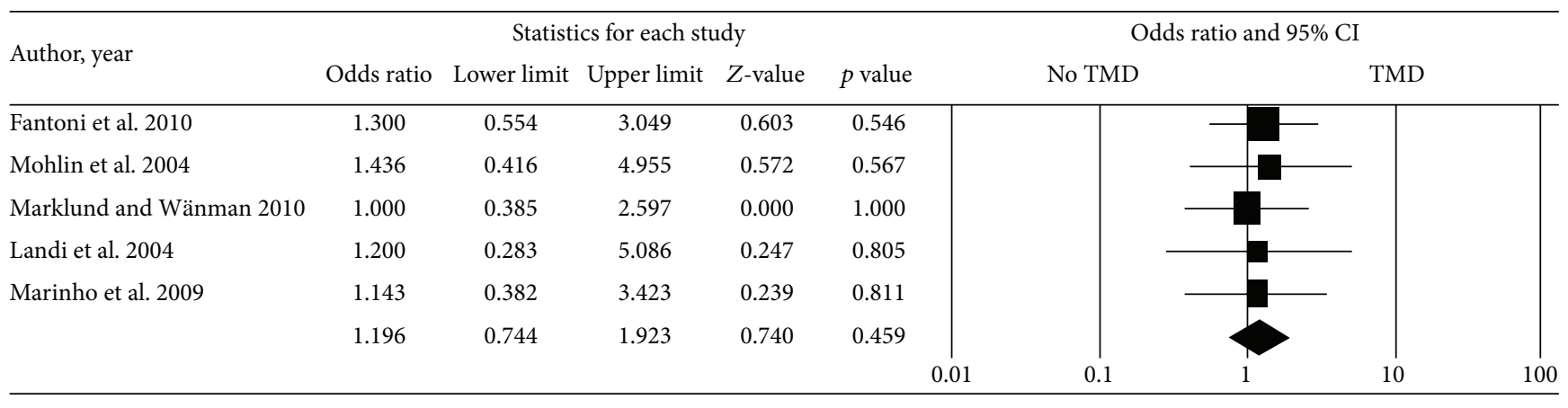

(g)

FIGURE 2: Forest plot estimate of the odds ratio for (a) laterotrusive interferences, (b) no canine guidance, (c) RCP-MI discrepancy $\geq 2$ mm, (d) mediotrusive interferences, (e) open-bite, (f) overbite, and (g) overjet.

the odds of TMD diagnosis (OR $=2.446,95 \%$ CI [1.573, 3.802], $p<0.001$, and $I^{2}=9.33 \%$ ) (Figure $2(\mathrm{~b})$ ) as did retruded contact position to maximal intercuspation discrepancies $\geq 2 \mathrm{~mm}$ (RCP-MI) $(\mathrm{OR}=1.668,95 \%$ CI [1.032, 2.695], $p=0.037$, and $I^{2}=12 \%$ ) (Figure 2(c)). Funnel plots for assessing the reporting were only provided for the analysis of canine guidance where the number of studies combined was at least three (Figure 3). In the absence of bias and heterogeneity, a funnel plot should resemble a symmetrically inverted funnel. If reporting or selection bias was present, such as unpublished smaller studies without statistically significant effects, this would lead to the asymmetrical appearance of a funnel plot. Overall pooled odds ratios for mediotrusive interferences $(\mathrm{OR}=1.396,95 \% \mathrm{CI}$ $[0.764,2.552], p=0.278$, and $I^{2}=69 \%$ ) (Figure $2(\mathrm{~d})$ ), openbite $\left(\mathrm{OR}=0.828,95 \%\right.$ CI $\left.[0.451,1.520], p=0.543, I^{2}=0 \%\right)$ (Figure 2(e)), overbite (OR $=0.809,95 \%$ CI $[0.544,1.202]$, $p=0.293$, and $I^{2}=11 \%$ ) (Figure $2(\mathrm{f})$ ), and overjet (OR = $1.196,95 \%$ CI $[0.744,1.923], p=0.459$, and $I^{2}=0 \%$ ) did not prove to be significant (Figure $2(\mathrm{~g})$ ). The combined analysis for mediotrusive interferences showed significant $\left(I^{2}=69 \%\right.$, $p=0.0119$ ) heterogeneity (Table 4 ).

\section{Discussion}

Our study found that static occlusal factors (overjet, overbite, open-bite, and crossbite) had no significant association with TMD. Of the dynamic occlusal factors assessed, only the absence of canine guidance, laterotrusive interferences, and
TABLE 4: Assessment of heterogeneity.

\begin{tabular}{lcccc}
\hline & $Q$-value & df & $p$ value & $I^{2}$ \\
\hline Laterotrusive interferences & 0.313 & 1 & 0.576 & $0 \%$ \\
Mediotrusive interferences $^{\dagger}$ & 8.835 & 3 & $0.032^{\dagger}$ & $66 \%$ \\
No canine guidance & 2.206 & 2 & 0.332 & $9 \%$ \\
Open-bite & 1.130 & 3 & 0.770 & $0 \%$ \\
Overbite & 4.476 & 4 & 0.345 & $11 \%$ \\
Overjet & 0.262 & 4 & 0.992 & $0 \%$ \\
RCP-MI $\geq 2 \mathrm{~mm}$ & 2.268 & 2 & 0.322 & $12 \%$ \\
\hline
\end{tabular}

${ }^{\dagger}$ Significant heterogeneity.

RCP-MI discrepancies $\geq 2$ mm demonstrated significant ORs when pooled across studies. Mediotrusive interferences did not show significance. These results suggest that dysfunctions of dynamic occlusion may act as risk factors for TMD.

It has been hypothesized that TMD arises from a necessary combination of both orthopedic instability surrounding the TMJ and loading of the joint itself [19]. Orthopedic instability is not synonymous with malocclusion. Some malocclusions are orthopedically stable, and therefore only malocclusions that disrupt the stomatognathic structure should be considered as risk factors for developing TMD. Static occlusal dysfunctions have the potential to act as risk factors only if they involve significant orthopedic deviations to achieve intercuspation. Our results appear to support the position that the human TMJ is able to adapt to small, static 


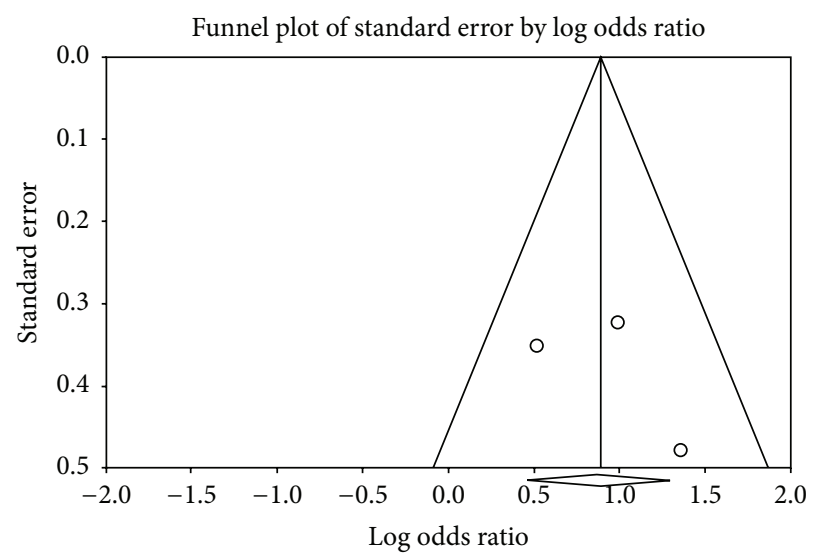

(a)

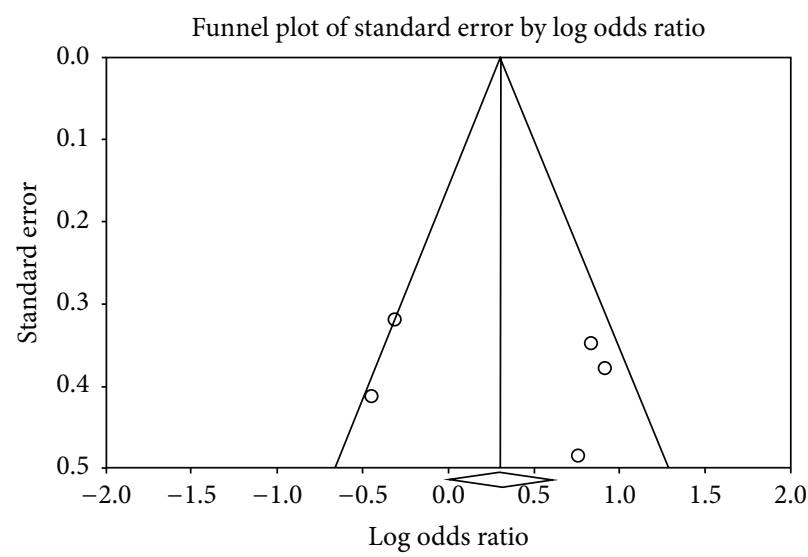

(c)

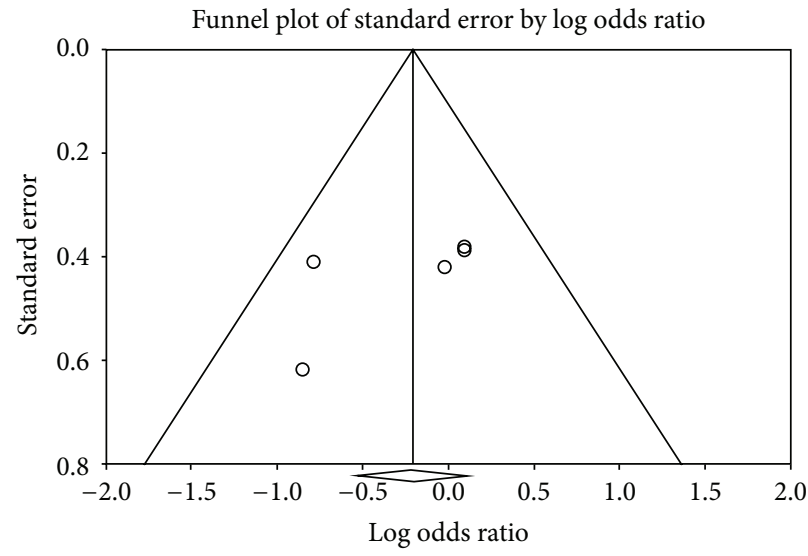

(e)

FIGURE 3: Funnel plot for the assessment of publication bias in interferences, (d) open-bite, (e) overbite, and (f) overjet.

occlusal discrepancies and can therefore tolerate considerable variation without showing signs of pathology.

Dynamic occlusal factors are of greater interest because of their potential to disrupt the TMJ. Our study found that laterotrusive interferences, the absence of canine guidance, and RCP-MI discrepancies $\geq 2 \mathrm{~mm}$ were associated with the presence of TMD. Lateral-side shifts of the mandible displace

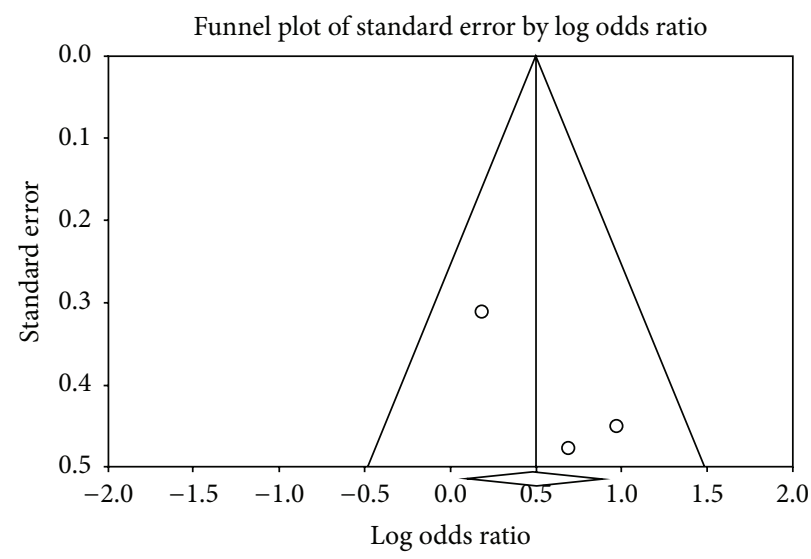

(b)

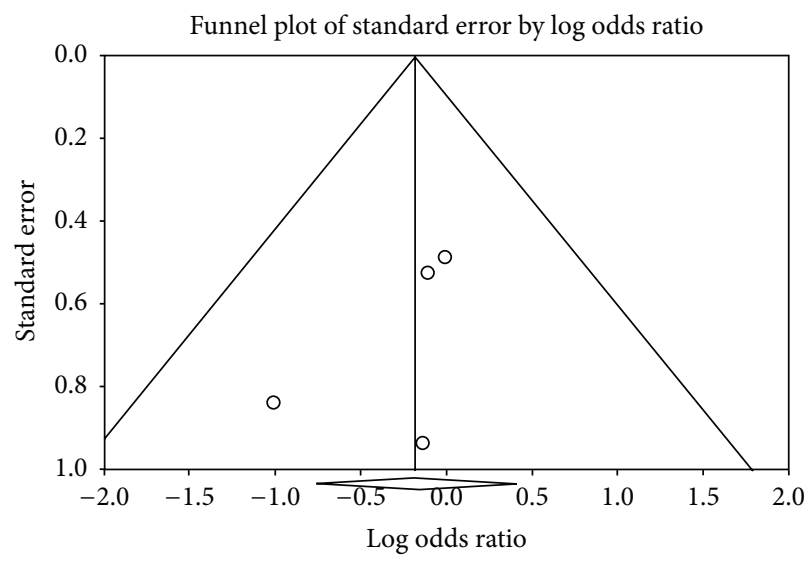

(d)

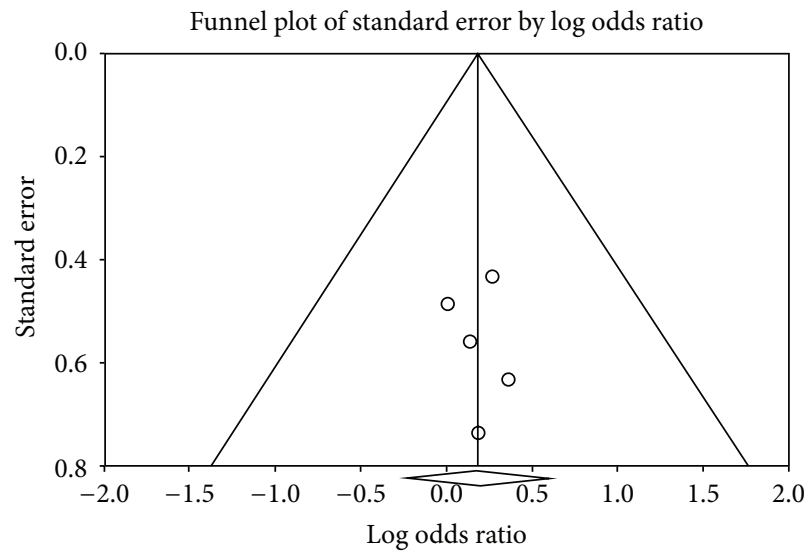

(f)

(a) no-canine guidance, (b) RCP-MI discrepancy, (c) mediotrusive 
studies. Canine guidance is protective as it disoccludes premolars and molars and avoids posterior loading during excursive mandibular movements. Likewise, RCP-MI discrepancies $\geq 2 \mathrm{~mm}$ represent the loss of a natural protective factor during mandibular excursion. Thus, the present findings of an association between dynamic occlusal factors and TMD may support at least in part the hypothesis that a stable occlusion is important to maintain the relationship between joint structures.

This analysis represents the first known effort to combine the quantitative results of multiple studies that used a similar TMD definition. The great variability in TMD disease definitions across the literature makes the synthesis of study findings difficult unless all studies included within an analysis share a similar definition. A limitation of our analysis was the paucity of comparable case/control studies defining TMD specifically as a dysfunction of myofascial pain. Our results for heterogeneity within each comparison should be interpreted with caution. In small meta-analyses such as this one, an incorrect zero between study variance estimates may be obtained, leading to a false assumption of homogeneity. Although many meta-analyses include between 2 and 4 studies, such a sample is often inadequate for accurately estimating heterogeneity.

The variety of RDC/TMD algorithms available is designed to encompass the entire spectrum of TMD disorders. Axis $1 \mathrm{RDC} / \mathrm{TMD}$ algorithms follow a dental mechanistic etiology and diagnose TMD with a clinical physical examination. Axis 2 algorithms follow a biopsychosocial etiology and diagnose TMD with a series of biobehavioral questionnaires. Each axis is further subdivided into a series of groups, each with their own diagnostic algorithm. By this way, the TMD diagnosis is assigned to a specific disorder. Without knowing the axis and group used, the TMD definition remains unknown to the reader. The primary difficulty in conducting this meta-analysis lies in the variety of TMD definitions that researchers used. This meta-analysis defined TMD as a muscle disorder, approximating an Axis 1 group 1. It was thought that the compounded forces generated by malocclusion would not be great enough to precipitate disc displacement (Axis 1 group 2). As a result, the majority of TMD experienced by patients with malocclusion would likely originate from muscular dysfunction. Despite our best efforts, the final 6 studies ultimately varied in the precise way they defined TMD whether it was by a muscular checklist, an RDC/TMD criterion, or a muscular/joint dysfunction on clinical examination. Not only did researchers use a variety of sometimes unvalidated diagnostic criteria, but also many researchers were not explicit in their TMD definitions. For example, Marinho used an RDC/TMD Axis 1 definition but did not specify the group algorithm. Likewise, Selaimen only mentioned that an RDC/TMD definition was used.

Because TMD is an umbrella term covering a variety of conditions, researchers should be aware that vague definitions make their results difficult to interpret clinically. The authors of this study were often left with the role of inferring crucial study details in order to assess the comparability among studies for inclusion. Ideally, TMD researchers should use the appropriate TMD/RDC axis and group that reflect their desired disease definition. Quality meta-analyses could then be conducted pooling those studies to yield clinically meaningful results. It should be noted that the updated DC/TMD criteria released in February 2014 were not available to the authors for use at the time of this metaanalysis. The RDC was introduced with the intension of future revision, and the revised DC improves on the validity and accuracy of RDC Axis 1 diagnostic category. Of note, the $\mathrm{DC} / \mathrm{TMD}$ Axis 1 group 1 diagnostic criterion is now a physical assessment of pain-related disorders and includes a questionnaire to screen for pain. Presently, case/control studies using this updated diagnostic criterion are not available.

Other sources of bias include blinding and confounding factors that were either unmentioned or unaccounted for. For instance, only 2 included studies $[12,14]$ explicitly mentioned examiner blinding in their methods. After carefully reviewing the methods of the remaining included studies, it was decided that another 3 studies [9, 11, 13] left open the possibility of blinding. This is cause for concern because some of the presumably unblinded studies reported several significant findings that were synthesized in this study's analysis. Additionally, orthodontic and TMD treatment are serious confounders that mask exposure and disease status, respectively. Ideally, a history of orthodontic treatment should be a criterion for exclusion when assessing malocclusion exposure. Selaimen et al. and Marklund et al. did not assess the history of orthodontic or TMD treatment in their subjects $[12,14]$, and although Mohlin did take note of orthodontic treatment history, it is unclear how or if that information factored into the study design [13]. Interestingly, Landi is a coauthor of Fantoni's study, which comes 6 years after Landi's own, and this may have biased Fantoni's findings, although their relationship is unclear. Authors should be explicit about both the orthodontic status of their subjects and the precautions taken, either through exclusion or matching, to control confounding variables.

Another limitation of our analysis lies in the exclusive use of unadjusted odds ratios (OR). There is no documented consensus about whether or how to synthesize adjusted findings [22]. To reduce heterogeneity, the simplest strategy would be including only unadjusted results in a pooled analysis. This approach, although the simplest, may not necessarily be appropriate. Because it has been proposed that TMD is multifactorial, the pairwise comparisons of this meta-analysis may be inadequate to address the etiology. It should be noted that because authors rounded their data, some log 95\% CI were asymmetric [22]. As a result, we needed to adjust some ORs and confidence interval limits by \pm 0.01 to make their values symmetric.

\section{Conclusions}

Quantitative results from the current study should be interpreted with caution. Our results suggest that dysfunctions of dynamic occlusion may act as risk factors for TMD.

Authors intending to conduct meta-analyses evaluating TMD should be aware that many studies in TMD research do not follow standardized diagnostic criteria, making it difficult to compare results in a quantitative analysis. This study 
encourages future TMD investigators to use and follow the appropriate updated TMD/DC diagnostic criteria. Authors of TMD research should be sure to clearly define and state the axis and group of the algorithm used.

\section{Conflict of Interests}

The authors declare that there is no conflict of interests regarding the publication of this paper.

\section{Acknowledgment}

This study was presented as an oral presentation at the 2015 IADR/AADR/CADR General Session in Boston, MA, where travel costs were supported through the AADR Bloc Travel Grant.

\section{References}

[1] J. A. McNamara Jr., D. A. Seligman, and J. P. Okeson, "Occlusion, Orthodontic treatment, and temporomandibular disorders: a review," Journal of Orofacial Pain, vol. 9, no. 1, pp. 73-90, 1995.

[2] D. A. Seligman and A. G. Pullinger, "The role of functional occlusal relationships in temporomandibular disorders: a review," Journal of Craniomandibular Disorders : Facial \& Oral Pain, vol. 5, no. 4, pp. 265-279, 1991.

[3] D. A. Seligman and A. G. Pullinger, "The role of intercuspal occlusal relationships in temporomandibular disorders: a review," Journal of Craniomandibular Disorders : Facial \& Oral Pain, vol. 5, no. 2, pp. 96-106, 1991.

[4] S. F. Dworkin and L. LeResche, "Research diagnostic criteria for temporomandibular disorders: review, criteria, examinations and specifications, critique," Journal of Craniomandibular Disorders: Facial \& Oral Pain, vol. 6, no. 4, pp. 301-355, 1992.

[5] S. F. Dworkin, “The OPPERA study: act one," The Journal of Pain, vol. 12, no. 11, pp. T1-T3, 2011.

[6] T. Henrikson, M. Nilner, and J. Kurol, "Symptoms and signs of temporomandibular disorders before, during and after orthodontic treatment," Swedish Dental Journal, vol. 23, no. 56, pp. 193-207, 1999.

[7] A. C. D. C. F. Conti, P. V. P. Oltramari, R. D. L. Navarro, and M. R. de Almeida, "Examination of temporomandibular disorders in the orthodontic patient: a clinical guide," Journal of Applied Oral Science, vol. 15, no. 1, pp. 77-82, 2007.

[8] J. Stockstill, C. S. Greene, S. Kandasamy, D. Campbell, and D. Rinchuse, "Survey of orthodontic residency programs: teaching about occlusion, temporomandibular joints, and temporomandibular disorders in postgraduate curricula," American Journal of Orthodontics and Dentofacial Orthopedics, vol. 139, no. 1, pp. 17-23, 2011.

[9] F. Fantoni, G. Chiappe, N. Landi, M. Romagnoli, and M. Bosco, "A stepwise multiple regression model to assess the odds ratio between myofascial pain and 13 occlusal features in 238 Italian women," Quintessence International, vol. 41, no. 3, pp. e54-e61, 2010.

[10] N. Landi, D. Manfredini, F. Tognini, M. Romagnoli, and M. Bosco, "Quantification of the relative risk of multiple occlusal variables for muscle disorders of the stomatognathic system," The Journal of Prosthetic Dentistry, vol. 92, no. 2, pp. 190-195, 2004.
[11] C. C. Marinho, F. L. G. Cruz, and F. P. P. Leite, "Influência de alguns fatores oclusais na prevalência das disfunções temporomandibulares," Revista de Odontologia da UNESP, vol. 38, pp. 280-285, 2009.

[12] S. Marklund and A. Wänman, "Risk factors associated with incidence and persistence of signs and symptoms of temporomandibular disorders," Acta Odontologica Scandinavica, vol. 68, no. 5, pp. 289-299, 2010.

[13] B. O. Mohlin, K. Derweduwen, R. Pilley, A. Kingdon, W. C. Shaw, and P. Kenealy, "Malocclusion and temporomandibular disorder: a comparison of adolescents with moderate to severe dysfunction with those without signs and symptoms of temporomandibular disorder and their further development to 30 years of age," The Angle Orthodontist, vol. 74, no. 3, pp. 319-327, 2004.

[14] C. M. Selaimen, J. C. Jeronymo, D. P. Brilhante, E. M. Lima, P. K. Grossi, and M. L. Grossi, "Occlusal risk factors for temporomandibular disorders," The Angle Orthodontist, vol. 77, no. 3, pp. 471-477, 2007.

[15] M. D. Costa, G. D. R. T. Froes Jr., and C. N. Santos, "Evaluation of occlusal factors in patients with temporomandibular joint disorder," Dental Press Journal of Orthodontics, vol. 17, no. 6, pp. 61-68, 2012.

[16] D. Gesch, O. Bernhardt, T. Kocher, U. John, E. Hensel, and D. Alte, "Association of malocclusion and functional occlusion with signs of temporomandibular disorders in adults: results of the population-based study of health in Pomerania," The Angle Orthodontist, vol. 74, no. 4, pp. 512-520, 2004.

[17] R. Pahkala and M. Qvarnström, "Can temporomandibular dysfunction signs be predicted by early morphological or functional variables?" European Journal of Orthodontics, vol. 26, no. 4, pp. 367-373, 2004.

[18] B. Turasi, A. Ari-Demirkaya, and S. Biren, "Comparison of increased overjet cases and controls: normative data for condylar positions," Journal of Oral Rehabilitation, vol. 34, no. 2, pp. 129-135, 2007.

[19] J. P. Okeson, "Etiology of functional disturbances in the masticatory system," in Management of Temporomandibular Disorders and Occlusion, J. Dolan, Ed., chapter 7, pp. 130-163, Elsevier Mosby, 6th edition, 2008.

[20] Y. Yang, M. Yatabe, M. Ai, and K. Soneda, "The relation of canine guidance with laterotrusive movements at the incisal point and the working side condyle," Journal of Oral Rehabilitation, vol. 27, no. 10, pp. 911-917, 2000.

[21] B.-Y. Huang, T. Whittle, C. C. Peck, and G. M. Murray, "Ipsilateral interferences and working-side condylar movements," Archives of Oral Biology, vol. 51, no. 3, pp. 206-214, 2006.

[22] C. I. Voils, J. L. Crandell, Y. Chang, J. Leeman, and M. Sandelowski, "Combining adjusted and unadjusted findings in mixed research synthesis," Journal of Evaluation in Clinical Practice, vol. 17, no. 3, pp. 429-434, 2011. 


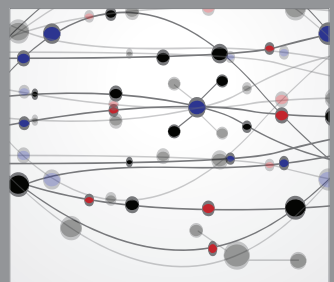

The Scientific World Journal
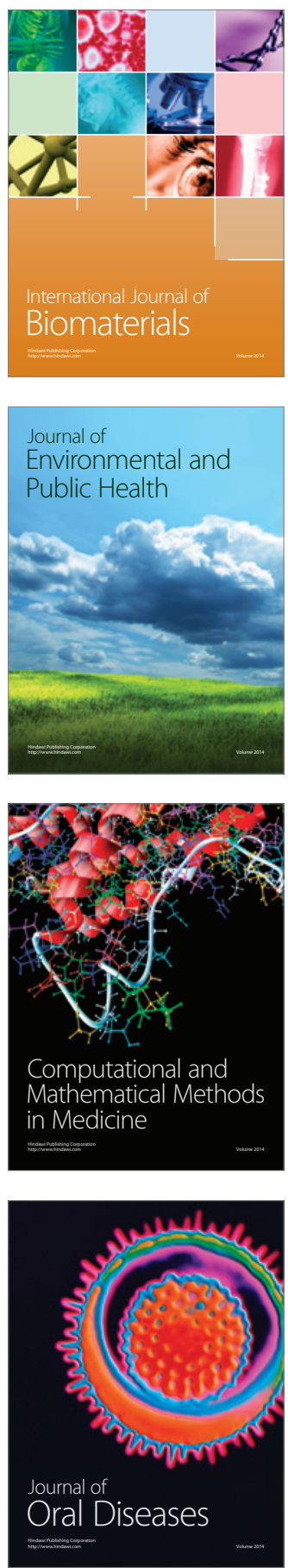
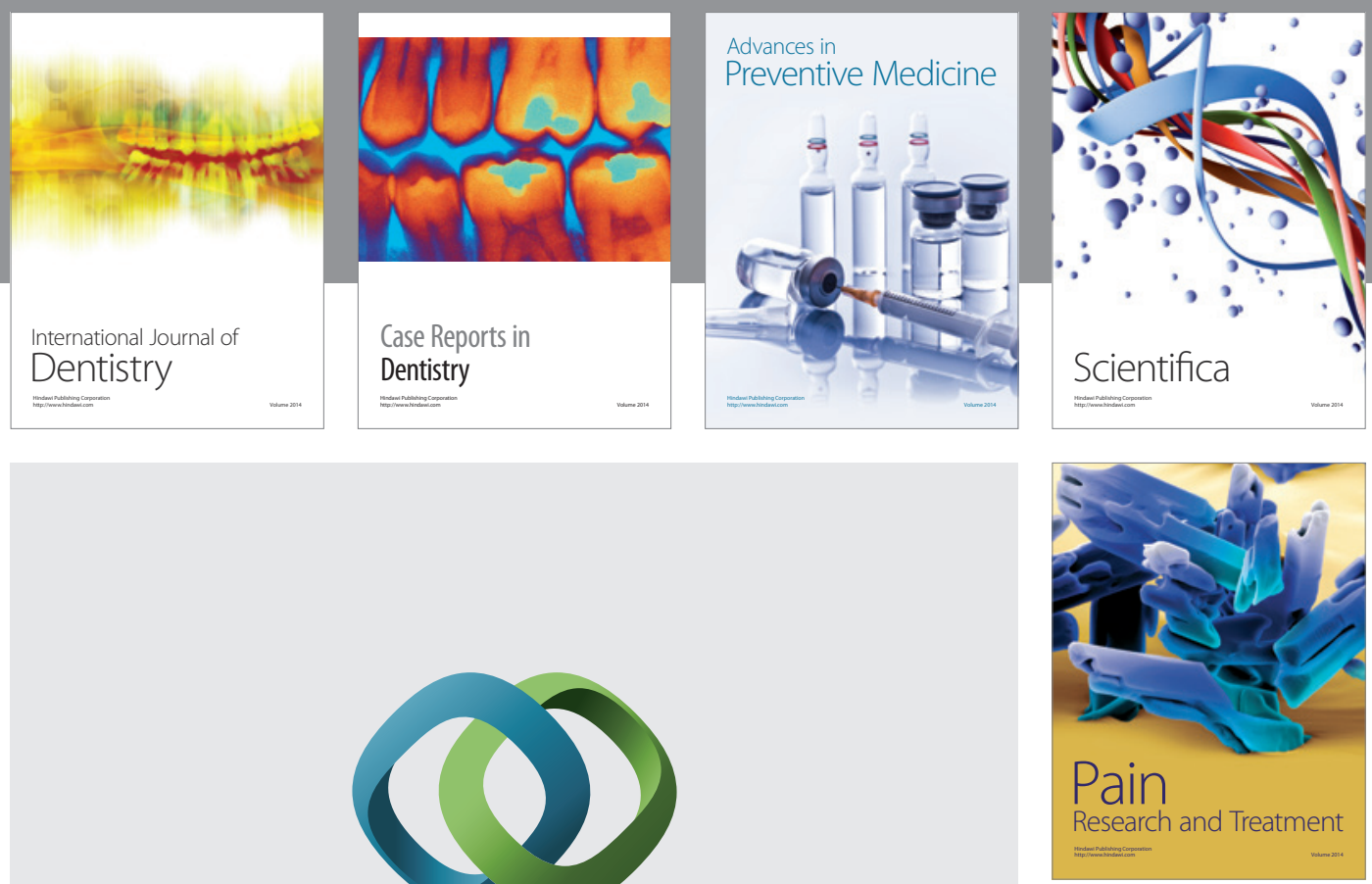

\section{Hindawi}

Submit your manuscripts at

http://www.hindawi.com
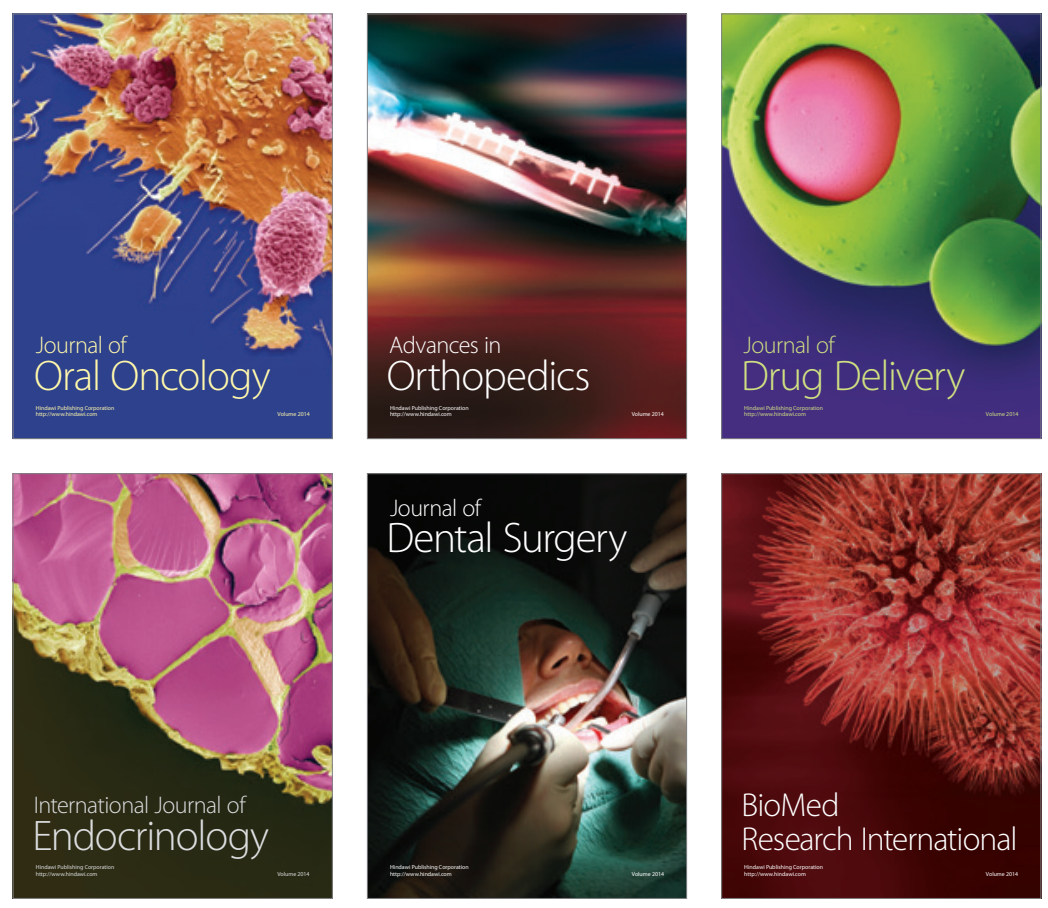

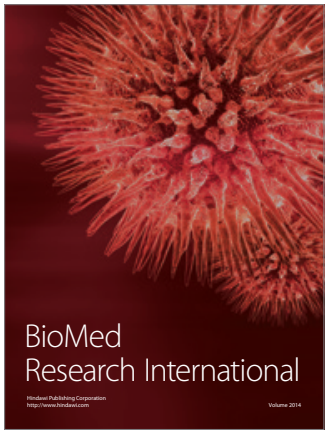

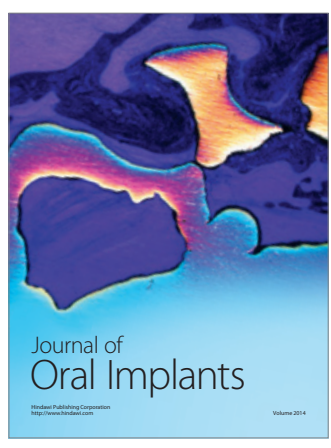
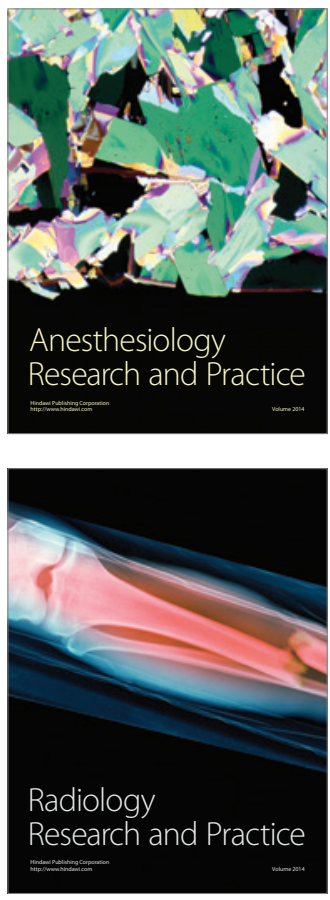